\title{
LXVII. On the transformation of elliptic functions
}

\section{Arthur Cayley Esq. M.A. F.C.P.S.}

To cite this article: Arthur Cayley Esq. M.A. F.C.P.S. (1845) LXVII. On the transformation of elliptic functions, Philosophical Magazine Series 3, 27:182, 424-427, DOI: $10.1080 / 14786444508646246$

To link to this article: http://dx.doi.org/10.1080/14786444508646246

曲 Published online: 30 Apr 2009.

Submit your article to this journal $₫$

山 Article views: 3

Q View related articles ¿ 


\section{[424]}

LXVII. On the Transformation of Elliptic Functions. By Arthur Cayley, Esq., M.A., F.C.P.S., Fellow of Trinity College, Cambridge*.

TN a former paper I gave a proof of Jacobi's theorem, which I I suggested would lead to the resolution of the very important problem of finding the relation between the complete functions. This is in fact effected by the formulæ there given, but there is an apparent indeterminateness in them, the cause of which it is necessary to explain, and which I shall now show to be inherent in the problem. For the sake of supplying an omission, for the detection of which I am indebted to $\mathrm{Mr}$. Bronwin, I will first recapitulate the steps of the demonstration.

If $\frac{1}{2} \omega, \frac{1}{2} v \dagger$ be the complete functions corresponding to $\varphi x$, then this function is expressible in the form

$$
\phi x=x \Pi\left(1+\frac{x}{m \omega+n v}\right) \div \Pi\left(1+\frac{x}{m+\frac{1}{2} \omega+n+\frac{1}{2} v}\right) \text {. }
$$

Let $p$ be any prime number, $\mu, \nu$ integers not divisible by $p$, and

The function

$$
\theta=\frac{\mu \omega+\nu u}{p}
$$

$$
\varphi_{1} x=\phi x \cdot \frac{\phi(x+2 \theta)}{\phi 2 \theta} \frac{\varphi(x+4 \theta)}{\phi 4 \theta} \ldots \frac{\phi(x+2(p-1) \theta)}{\phi(2(p-1) \theta)}
$$

is always reducible to the form

$$
x \Pi\left(1+\frac{x}{m^{\prime} \omega^{\prime}+n^{\prime} v^{\prime}}\right) \div \Pi\left(1+\frac{x}{m^{\prime}+\frac{1}{2} \omega^{\prime}+n^{\prime}+\frac{1}{2} v^{\prime}}\right) \text {. }
$$

Or $\phi_{1} x$ is an inverse function, the complete functions of which are $\frac{1}{2} \omega^{\prime}, \frac{1}{2} v^{\prime}$. And where $\omega^{\prime}, v^{\prime}$ are connected with $\omega, v$ by the equations

$$
\begin{aligned}
& w^{\prime}=\frac{1}{p}(\alpha \omega+b u), \\
& v^{\prime}=\frac{1}{p}\left(\alpha^{\prime} \omega+b^{\prime} u\right),
\end{aligned}
$$

$\alpha, \varepsilon, \alpha^{\prime}, \sigma^{\prime}$ being any integers subject to the conditions that $\alpha, b^{\prime}$ are odd and $\alpha^{\prime}, b$ even; also

* Communicated by the Author.

+ Analogous to the $\mathbf{K}, \mathbf{K}^{\prime}$ เ of M. Jacobi. 


$$
\begin{aligned}
& \alpha b^{\prime}-\alpha^{\prime} b=p, \\
& \mu b^{\prime}-\nu \alpha^{\prime}=l^{\prime} p_{,} \\
& \mu \sigma-\nu \alpha=l p,
\end{aligned}
$$

$l, l^{\prime}$ being any integers whatever. In fact, to prove this, we have only to consider the general form of a factor in the numerator of $\varphi_{1} x$. Omitting a constant factor, this is

$$
\left(1+\frac{x}{m \omega+n v+2 r \theta}\right) \quad[r<p] \text {; }
$$

and it is to be shown that we can always satisfy the equation

$$
m \omega+n v+2 r \theta=m^{\prime} \omega^{\prime}+n^{\prime} v^{\prime},
$$

or the equations

$$
\begin{aligned}
p m+2 r \mu & =m^{\prime} \alpha+n^{\prime} \alpha^{\prime}, \\
p n+2 r \nu & =m^{\prime} \sigma+n^{\prime} \xi^{\prime} ;
\end{aligned}
$$

and also that to each set of values of $m, n, r$, there is a unique set of values of $m^{\prime}, n^{\prime}$, and vice versâ. "This is done in the paper referred to. Moreover, with the suppositions just made as to the numbers $\alpha, b^{\prime}$ being odd and $\alpha^{\prime}, b^{2}$ even, it is obvious that $m^{\prime}$ is odd or even, according as $m$ is, and $n^{l}$ according as $n$ is, which shows that we can likewise satisfy

$$
\overline{m+\frac{1}{2}} \omega+\overline{n+\frac{1}{2}} v+2 r \theta=\overline{m^{\prime}+\frac{1}{2}} \omega^{\prime}+\overline{n^{\prime}+\frac{1}{2}} v^{\prime} ;
$$

and thus the denominator of $\phi_{1} x$ is also reducible to the required form.

Now proceeding to the immediate object of this paper, $\alpha, b$, $\alpha^{\prime}, b^{\prime}$, and consequently $\omega^{\prime}, v^{\prime}$ are to a certain extent indeterminate. Let $\mathrm{A}, \mathrm{B}, \mathrm{A}^{\prime}, \mathrm{B}^{\prime}$ be a particular set of values of $\alpha$, $\varepsilon, \alpha^{\prime}, \epsilon^{\prime}$, and $\mathrm{O}, \mathrm{P}$ the corresponding values of $\omega^{\prime}, v^{\prime}$. We have evidently $A, B^{\prime}$ odd and $A^{\prime}, B$ even. Also

$$
\begin{aligned}
& A B^{\prime}-A^{\prime} B=p, \\
& \mu B^{\prime}-\nu A^{\prime}=L^{\prime} p, \\
& \mu \mathrm{B}-\nu \mathrm{A}=\mathrm{L} p, \\
& \mathrm{O}=\frac{1}{p}(\mathrm{~A} \omega+\mathrm{B} v), \\
& \mathrm{U}=\frac{1}{p}\left(\mathrm{~A}^{\prime} \omega+\mathrm{B}^{\prime} v\right) .
\end{aligned}
$$

By eliminating $w, v$ from these equations and the former system, it is easy to obtain

$$
\begin{aligned}
& \omega^{\prime}=a \mathrm{O}+b \mathrm{U}, \\
& v^{\prime}=a^{\prime} \mathrm{O}+b^{\prime} \mathrm{U},
\end{aligned}
$$

Phil. Mag. S. 3. Vol.27. No. 182. Dec. 1845. 2 F 
where

$$
\begin{aligned}
a & =\frac{1}{p}\left(\alpha \mathrm{B}^{\prime}-6 \mathrm{~A}^{\prime}\right), & b & =-\frac{1}{p}(\alpha \mathrm{B}-\varepsilon \mathrm{A}), \\
a^{\prime} & =\frac{1}{p}\left(\alpha^{\prime} \mathrm{B}^{\prime}-b^{\prime} \mathrm{A}^{\prime}\right), & b^{\prime} & =-\frac{1}{p}\left(\alpha^{\prime} \mathrm{B}-b^{\prime} \mathrm{A}\right) .
\end{aligned}
$$

The coefficients $a, b, a^{\prime}, b^{\prime}$ are integers, as is obvious from the equation $\mu\left(\alpha \mathrm{B}^{\prime}-6 \mathrm{~A}^{\prime}\right)=p\left(\mathrm{~L}^{\prime} \alpha-l \mathrm{~A}^{\prime}\right)$, and the others analogous to it; moreover, $a, b^{\prime}$ are odd and $a^{\prime}, b$ are even, and

i. e.

$$
a b^{\prime}-a^{\prime} \cdot b=\frac{1}{p^{2}}\left(\mathbf{A} B^{\prime}-A^{\prime} \mathrm{B}\right)\left(\alpha b^{\prime}-\alpha^{\prime} b\right) \text {; }
$$

Hence the theorem, - The general values $w^{\prime}, v^{\prime}$ of the complete functions are linearly connected with the particular system of values $\mathrm{O}, \mathrm{U}$ by the equations, $\omega^{\prime}=a \mathrm{O}+b \mathrm{U}$, $v=a^{\prime} \mathrm{O}+b^{\prime} \mathrm{U}$, in which $a, b^{\prime}$ are odd integers and $a^{\prime}, b$ even ones, satisfying the condition $a b^{\prime}-a^{\prime} b=1$. ."

With this relation between $\mathrm{O}, \mathrm{U}$ and $\omega^{\prime}, v^{\prime}$, it is easy to show that the function $\phi_{1} x$ is precisely the same, whether $O$, $\mathrm{U}$ or $\omega^{\prime}, v^{\prime}$ be taken for the complete functions. In fact, stating the proposition relatively to $\varphi x$, we have, - "The inverse function $\varphi x$ is not altered by the change of $\omega, v$ into $\omega^{\prime}, v^{\prime}$, where $\omega^{\prime}=\alpha \omega+f v, v^{\prime}=\alpha^{\prime} \omega+f^{\prime} v$, and $\alpha, b, \alpha^{\prime}, f^{\prime}$ satisfy the conditions that $\alpha, f^{\prime}$ are odd, $\alpha^{\prime}, f$ even, and $\alpha b^{\prime}-\alpha^{\prime} b=1$." This is immediately shown by writing

or

$$
\begin{aligned}
& m \omega+n v=m^{\prime} \omega^{\prime}+n^{\prime} v^{\prime}, \\
& m=m^{\prime} \alpha+n^{\prime} \alpha^{\prime}, \\
& n=m^{\prime} G+n^{\prime} \sigma^{\prime} .
\end{aligned}
$$

It is obvious that to each set of values of $m, n$ there is a unique set of values of $m^{\prime}, n^{\prime}$, and vice vers $a$ : also that odd or even values of $m, m^{\prime}$ or $n, n^{\prime}$ always correspond to each. It is, in fact, the preceding reasoning applied to the case of $p=1$.

Hence finally the theorem, - The only conditions for determining $\omega^{\prime}, v^{\prime}$ are the equations

$$
\omega^{\prime}=\frac{1}{p}\left(\alpha \omega+\sigma_{v}\right), \quad v^{\prime}=\frac{1}{p}\left(\alpha^{\prime} \omega+\sigma^{\prime} v\right),
$$

where $\alpha, \sigma^{\prime}$ are odd and $\alpha^{\prime}, \epsilon_{\text {even, and }}$

$$
\alpha b^{\prime}-\alpha^{\prime} b=p, \quad \mu b^{\prime}-\nu \alpha^{\prime}=l^{\prime} p, \quad \mu b-\nu \alpha=l p,
$$

$l$ and $l$ arbitrary integers: and it is absolutely indifferent what system of values is adopted for $\omega^{\prime}, v^{\prime}$, the value of $\varphi_{1} x$ is precisely the same."

We derive from the above the somewhat singular conclusion, that the complete functions are not absolutely determi- 
nate functions of the modulus; notwithstanding that they are given by the apparently determinate conditions,

$$
\begin{aligned}
& \frac{1}{2} \omega=\int_{0}^{\frac{1}{c}} \frac{d x}{\sqrt{\left(1-c^{2} x^{2}\right)\left(1+e^{2} x^{2}\right)}}, \\
& \frac{1}{2} v=\int_{0}^{\frac{1}{e}} \frac{d x}{\sqrt{\left(1+c^{2} x^{2}\right)\left(1-e^{2} x^{2}\right)}} .
\end{aligned}
$$

In fact definite integrals are in many cases really indeterminate, and acquire different values according as we consider the variable to pass through real values, or through imaginary ones. Where the limits are real, it is tacitly supposed that the variable passes through a succession of real values, and thus $w, v$ may be considered as completely determined by these equations, but only in consequence of this tacit supposition. If $c$ and $e$ are imaginary, there is absolutely no system of values to be selected for $\omega, v$ in preference to any other system. The only remaining difficulty is to show from the integral itself, independently of the theory of elliptic functions, that such integrals contain an indeterminateness of two arbitrary integers; and this difficulty is equally great in the simplest cases. Why, à priori, do the functions

$$
\sin ^{-1} x=\int_{0} \frac{d x}{\sqrt{1-x^{2}}} \quad \text { or } \log x=\int_{0} \frac{d x}{x}
$$

contain a single indeterminate integer ?

Obs. I am of course aware, that in treating of the properties of such products as $\Pi\left(1+\frac{x}{m \omega+n v}\right)$, it is absolutely necessary to pay attention to the relations between the infinite limiting values of $m$ and $n$; and that this introduces certain exponential factors, to which no allusion has been made. But these factors always disappear from the quotient of two such products, and to have made mention of them would only have been embarrassing the demonstration without necessity.

LXVIII. On the Diurnal Changes of the Aqueous Portion of the Atmosphere, and their Effects on the Barometer. By Thomas Hopkins, Esq.*

I $T$ is admitted by meteorologists, that the various quantities 1 of aqueous vapour which exist in the atmosphere during the different hours of the day, contribute to the production of

* Communicated by the Author; having been read at the Manchester Literary and Philosophical Society, October 7, 1845. 